\title{
Анализ орбитальных теорий для построения численной теории физической либрации Луны
}

\author{
A.А. Загидуллин ${ }^{1}$, H.К. Петрова ${ }^{1,2}$, В.С. Усанин ${ }^{1}$, Ю.А. Нефедьев ${ }^{1}$ \\ 1 Казанский федеральный университет, Кремлевская, 18, Казань, Россия, 420008 \\ arhtur.zagidullin@ya.ru,Vladimir.Usanin@kpfu.ru,Yura.Nefedyev@gmail.com \\ 2 Казанский государственный энергетический университет, Красносельская, 51, Казань, Россия, 420066 \\ nk_petrova@mail.ru
}

Поступила в редакцию 14 ноября 2017 г.

\begin{abstract}
Аннотация. При построении численной теории физической либрации перед нами встала задача сравнения численной и аналитической теорий орбитального движения Луны. В ходе исследования нам было важно понять, как различия в точности описания движения центра масс скажутся на поведении либрации. На данный момент мы представляем результаты первого этапа сравнения, а именно: описание поведения во времени различий между двумя типами орбитальных теорий. Нами была использована аналитическая теория Гутцвиллера и Шмидта, построенная в рамках главной проблемы, и современная численная теория DE431 JPL NASA, учитывающая большое количество факторов, выходящих за рамки главной проблемы, которые было бы сложно или даже невозможно учесть при получении аналитического решения. Произведя необходимые редукции при приведении обеих теорий к единой системе отсчета, мы сравнили оба решения на интервале в 800 лет. В итоге мы получили, что амплитуда в долготе за этот период не превосходит 80 угловых секунд, а в широте 10 угловых секунд. Основным источником расхождений являются эффекты, не учитываемые в главной проблеме, такие как: планетные возмущения, движение плоскости эклиптики, сжатие Земли, приливные эффекты и эффекты общей теории относительности.

ANALYSIS OF ORBITAL THEORIES FOR THE CONSTRUCTION OF THE NUMERICAL THEORY OF THE LUNAR PHYSICAL LIBRATIONS, by A.A. Zagidullin, N.K. Petrova, V.S. Usanin, $Y$.A. Nefedyev. The problem of comparing the numerical and analytical theories of the orbital motion of the Moon came before us at the stage of constructing a numerical theory of physical libration. In the course of the study, it was important for us to understand how the differences in the accuracy of the description of the center of mass motion will affect the behavior of the libration. Now we present the results of the first stage of the comparison, namely: the description of the time behavior of differences between the two types of orbital theories. We work with the analytical theory by Gutzwiller and Schmidt built in the framework of the main problem, and the modern numerical theory DE431 by JPL NASA, which takes into account a large number of factors that go beyond the main problem and which would be difficult or even impossible to take into account when obtaining an analytical solution. Having made the necessary reductions when we brought both theories to a unified reference frame, we compared both solutions on an interval of 800 years. As a result, we have obtained that the amplitude in longitude for this period does not exceed 80 arcseconds, and in latitude 10 arcseconds. The main sources of discrepancies are perturbations from planets and asteroids, motion of the ecliptic plane, the Earth's figure, tidal effects, and general relativity in numerical ephemerides, which were not included in analytical theory.
\end{abstract}

Ключевые слова: движение Луны, аналитический и численный подходы 


\section{1 Введение}

Исследование физики и динамики Луны актуально в связи с планами ее освоения в ближайшем будущем. В Казанском университете исследования Луны являются традиционными (Петрова и др., 2013). Разработка теории физической либрации - это одно из направлений изучения нашего спутника. Аналитическая теория физической либрации Луны (ФЛЛ) была построена (Петрова, 1996) с точностью $0^{\prime \prime} .01$ на основе аналитической теории орбитального движения Луны (Гутцвиллер, Шмидт, 1986). В настоящее время нами разрабатывается численный подход в теории ФЛЛ, и в качестве первого этапа мы построили (Загидуллин и др., 2016) численную копию аналитической теории (Петрова, 1996), то есть численную теорию в рамках главной проблемы, рассматривая орбитальное и вращательное движение независимыми, используя ту же орбитальную теорию. Проблемы начались, когда мы стали переходить на численную эфемериду DE431 (Фолкнер и др., 2014) для описания движения центра масс: расхождения с теорией (Петрова, 1996) в значениях углов ФЛЛ уже в начале интегрирования были более $200^{\prime \prime}$, и из-за наличия векового тренда достигали более $2000^{\prime \prime}$ уже через год. Причиной такого поведения остаточных разностей мог быть и некорректный переход к динамическим эфемеридам, и влияние тех эффектов, которые не были учтены в теории (Гутцвиллер, Шмидт, 1986). Очевидно, что физическая либрация Луны очень чувствительна к используемой орбитальной теории и поэтому анализ орбитальных теорий необходим для того, чтобы, во-первых, обеспечить корректность применения теории, во-вторых, оценить влияние на вращение Луны тех тонких эффектов, что отличают современные теории от модели главной проблемы. При переходе от аналитических орбитальных теорий к динамическим эфемеридам необходимо понимать специфику построения теории. Понимание всех тонкостей в орбитальных теориях является неотъемлемой частью для получения точной теории вращения Луны.

\section{2 Описание орбитальных теорий}

Аналитическая теория (Гутцвиллер, Шмидт, 1986) построена в рамках главной проблемы и решена методом Хилла-Брауна, адаптированным для вычислений на компьютере У. Эккертом. Главная проблема рассматривает гравитационное взаимодействие только трех точечных тел - Луны, Земли и Солнца. В той же работе выполнен хороший обзор исторического развития решения проблемы в применении к этим трем телам. В качестве системы отсчета в теории выбрана эклиптическая система координат. Авторы теории оценивали максимальную ошибку каждого ее слагаемого величиной 2 см в радиальном направлении и 20 см в других направлениях.

Численные динамические эфемериды серии DE построены на основе постньютоновского формализма. Данная модель учитывает ускорения, вызванные приливными деформациями Земли, формой Земли и Луны, а также модель лунной либрации. Уравнения строятся для планет всей Солнечной системы, поэтому в каждой орбите учтены косвенные влияния от других планет, а также главного пояса астероидов и пояса Койпера. В качестве системы отсчета выбирается квазиинерциальная ICRF. DE432, построенная в помощь проекту New Horizons, незначительно отличается от DE430/431. Haчиная с DE430 астрономическая единица зафиксирована на значении 149597870.700 км, а до DE430 вычислялась на основе гауссовой постоянной. Динамическая модель DE430 содержит возмущения, вызванные взаимодействием на границе между ядром и нижней мантией, которые вызывают диссипацию. Поскольку неизвестны с необходимой точностью параметры ядра, то динамическая теория DE430 имеет хорошую точность только на интервале от 1550 до 2650 года. Разность между орбитами Луны DE430 и 431 обусловлена точностью лазерной локации и не превышает 1 м. 


\section{3 Преобразование системы отсчета и учет главной проблемы}

Начнем с преобразования системы отсчета динамической теории DE. Поскольку динамическая теория строится в экваториальной невращающейся системе отсчета, то первым этапом является переход в эклиптическую систему координат, а затем переход во вращающуюся систему координат. Переход от экваториальной системы координат к эклиптической эпохи J2000.0 осуществляется поворотом на фиксированной угол $\varepsilon_{0}=84381^{\prime \prime} .448$ :

$$
\left(\begin{array}{l}
x \\
y \\
z
\end{array}\right)_{\text {ecliptic }}=R_{x}\left(\varepsilon_{0}\right) \cdot\left(\begin{array}{l}
x \\
y \\
z
\end{array}\right)_{\text {equator }} .
$$

Средняя долгота Луны при переходе во вращающуюся систему координат будет:

$$
\bar{L}_{\text {sideric }}=F+\Omega-p_{A},
$$

где $F$ - это средний аргумент широты Луны, входящий в число аргументов Делоне (представляемый в виде полинома по времени), $p_{A}$ - общая прецессия в долготе, $\Omega$ - средняя долгота восходящего узла орбиты Луны на эклиптике.

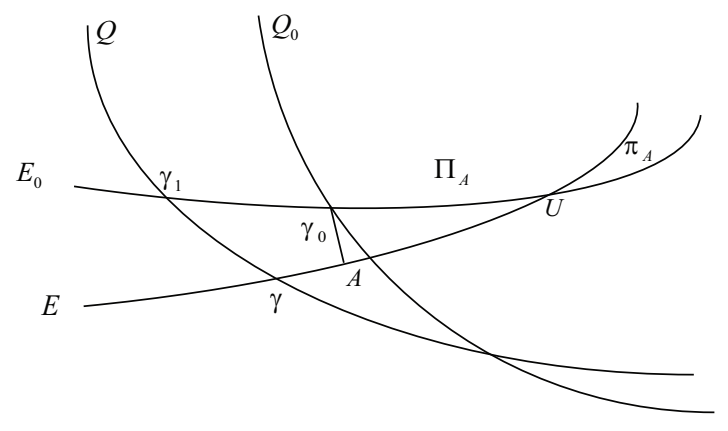

Рис. 1. Система подвижного и неподвижного экватора и эклиптики, где $Q_{0}, E_{0}$ обозначают неподвижный экватор и эклиптику на эпоху $\mathrm{J} 2000.0$ соответственно, $Q, E$ обозначают подвижный экватор и эклиптику на дату, точка $A$ выбрана так, чтобы $U A=U \gamma_{0}$

Теперь рассмотрим преобразование системы отсчета Шмидта. В теории Шмидта не учитывается прецессия от планет, то есть изменение орбиты Земли относительно Солнца. Поэтому не было ясно, близка ли базовая плоскость этой теории к эклиптике текущей даты или к эклиптике фиксированной эпохи J2000.0. Анализ орбитальной теории Шмидта показал, что если мы будем принимать в качестве базовой плоскости эклиптику эпохи J2000.0, то разница в широте за интервал 800 лет достигает примерно 400", что составляет угол между плоскостями эклиптики текущей даты и эклиптики J2000.0. Таким образом, мы приходим к выводу, что базовой плоскостью системы Шмидта является эклиптика даты, а не эклиптика J2000.0. Для того чтобы учесть прецессию от планет, мы будем использовать систему уравнений подвижной эклиптики, которая описывается тремя углами: общая прецессия в долготе $-p_{A}$, угол наклона подвижной эклиптики к неподвижной $-\pi_{A}$ и долгота восходящего узла подвижной эклиптики на неподвижной - $\Pi_{A}$ (см. рис. 1).

Поскольку дифференциальные уравнения ФЛЛ (Петрова, 1996) записаны в системе координат с фиксированной эклиптикой J2000.0 в качестве базовой плоскости, вращающейся с угловой скоростью $n=\frac{d \bar{L}_{\text {sideric }}}{d t}$, то мы будем преобразовывать систему Шмидта к этой системе координат следующим образом. 

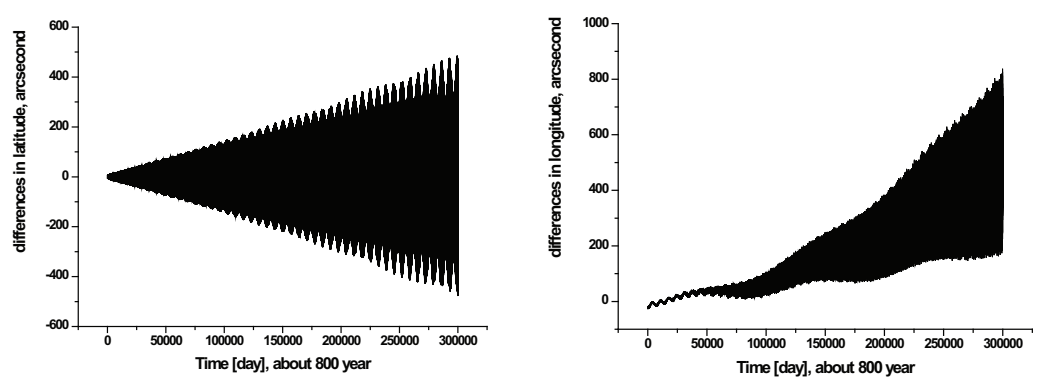

Рис. 2. Разности между теориями Шмидта и DE431 без учета векового ускорения и прецессии от планет, слева приведена разность в широте, справа - в долготе
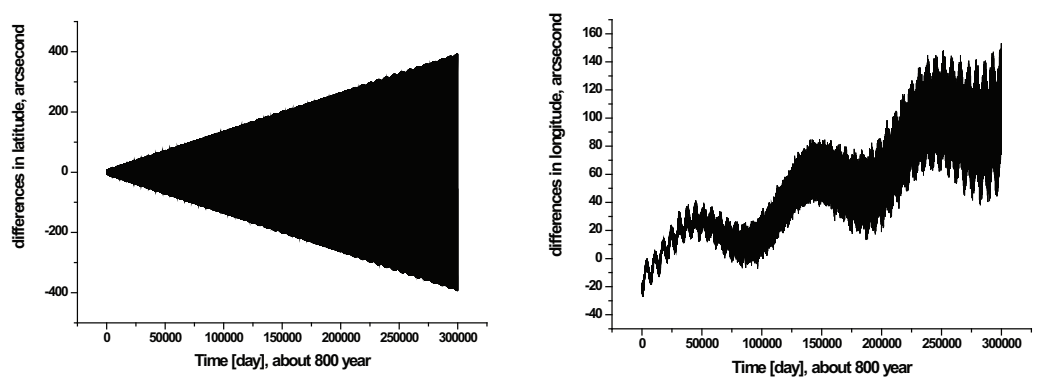

Рис. 3. Учет векового ускорения, слева приведена разность в широте, справа - в долготе
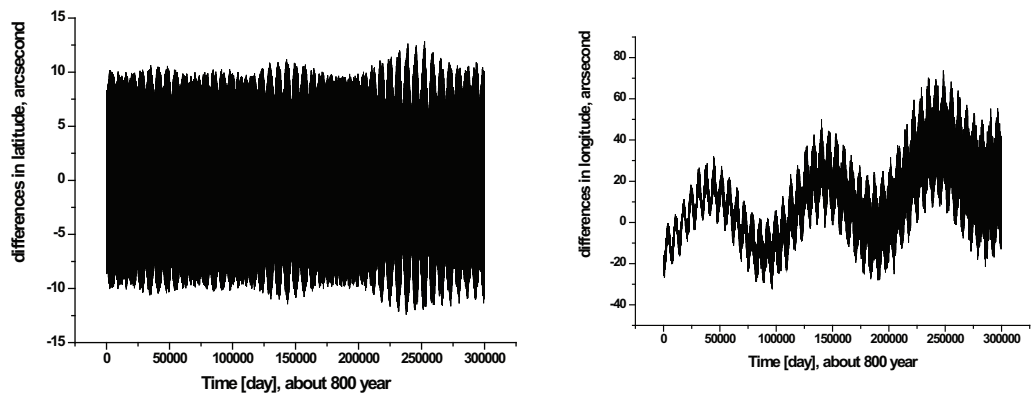

Рис. 4. Учет совместного влияния векового ускорения и прецессии от планет, слева приведена разность в широте, справа - в долготе

Перейдем в систему координат, где долготы отсчитываются от равноденствия даты, путем прибавления тропической средней долготы Луны к долготе Шмидта, поскольку система отчета у Шмидта определяется эклиптикой даты:

$$
L_{1}=L_{\text {Schmidt }}+\bar{L}_{\text {tropic }}=L_{\text {Schmidt }}+F+\Omega
$$

Перейдем от эклиптики даты к эклиптике эпохи, учтя прецессию от планет (Абалакин, 1979):

$$
r^{\prime}=R_{z}\left(-\Pi_{A}\right) \cdot R_{x}\left(-\pi_{A}\right) \cdot R_{z}\left(\Pi_{A}+p_{A}\right) \cdot r
$$


где $r=\left(\begin{array}{c}\cos B_{\text {Schmidt }} \cdot \cos L_{1} \\ \cos B_{\text {Schmidt }} \cdot \sin L_{1} \\ \sin B_{\text {Schmidt }}\end{array}\right), r^{\prime}=\left(\begin{array}{c}\cos B_{1} \cdot \cos L_{2} \\ \cos B_{1} \cdot \sin L_{2} \\ \sin B_{1}\end{array}\right)$.

Перейдем ко вращающимся осям координат, вычтя теперь сидерическую среднюю долготу Луны:

$$
L_{3}=L_{2}-\bar{L}_{\text {sideric }}=L_{2}-\left(F+\Omega-p_{A}\right) .
$$

Стоит отметить, что на больших промежутках времени нам необходимо учитывать также нелинейные слагаемые в разложении аргументов Делоне, поскольку за 800 лет в долготе накапливается эффект от векового ускорения Луны примерно $\frac{26^{\prime \prime} \cdot 8^{2}}{2}=832^{\prime \prime}$.

Проделав вышеуказанные преобразования для главной проблемы, мы получили результаты, показанные на рис. 2-4. Видно, что вековое ускорение влияет в большей степени на долготу, а прецессия от планет - на широту. Совместный учет обоих факторов уменьшает тренд разности в долготе с сотен до десятков секунд и практически устраняет нарастание со временем амплитуды разности в широте.

\section{4 Эффекты, не учитываемые в главной проблеме}

После учета прецессии и векового ускорения, разности остались велики. По долготе очень четко выделяется квазирезонансное слагаемое с периодом, близким к 273 годам. Согласно (Шапрон-Тузе, Шапрон, 2002), возмущения со стороны Венеры на орбиту Луны вызывают колебания именно с таким периодом и амплитудой примерно $14^{\prime \prime}$ :

$$
\begin{gathered}
L_{4}=L_{3}+14^{\prime \prime} .24883 \sin \left(18 V-16 T-l+26^{\circ} .54\right), \\
B_{2}=B_{1}+0^{\prime \prime} .63037 \sin \left(18 V-16 T-l+F+26^{\circ} .54\right)+0^{\prime \prime} .63014 \sin \left(18 V-16 T-l-F+26^{\circ} .54\right),
\end{gathered}
$$

где $V$ - долгота Венеры и $T$ - долгота Земли были взяты из работы (Симон и др., 1994), $l$ - средняя аномалия Луны, один из аргументов Делоне.

Следующим значительным вкладом в остаточные разности является эффект от сжатия Земли на орбиту Луны (особенно на широтные составляющие) с амплитудой $7^{\prime \prime}-8^{\prime \prime}$ :

$$
\lambda=L_{4}+7^{\prime \prime} .06304 \sin (\zeta-F), \beta=B_{2}-8^{\prime \prime} .04508 \sin (\zeta), \zeta=F+\Omega,
$$

где $\zeta$ обозначает тропическую среднюю долготу Луны.
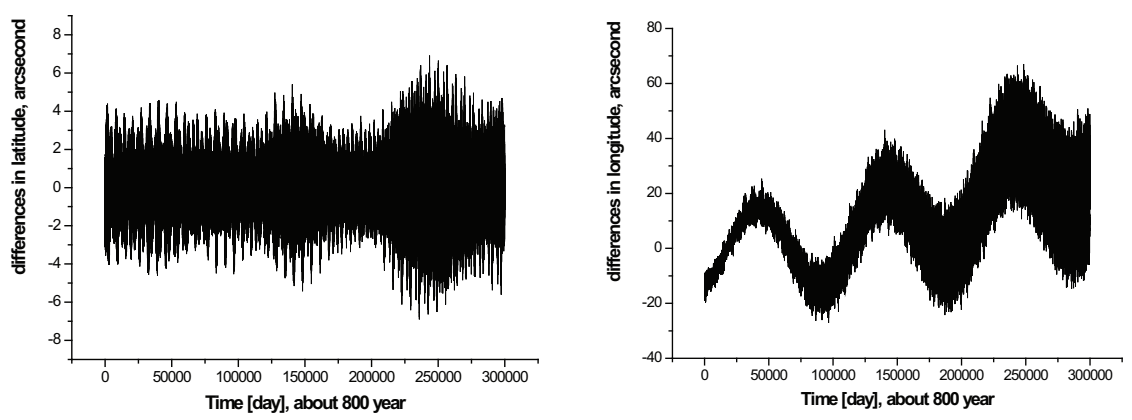

Рис. 5. Учет сжатия Земли, слева приведена разность в широте, справа - в долготе

В табл. 1 приведены все числовые значения коэффициентов используемых рядов. Они взяты из работы (Пти, Лузум, 2010), кроме $\Pi_{A}$ и $\pi_{A}$, которые были взяты из работы (Лиске и др., 1977). Однако существует неоднозначность в значениях некоторых аргументов. Вклад этих величин приводит к расхождению на величину, не превосходящую $2^{\prime \prime}$. На рис. 5 и 6 видно, что возмущения 

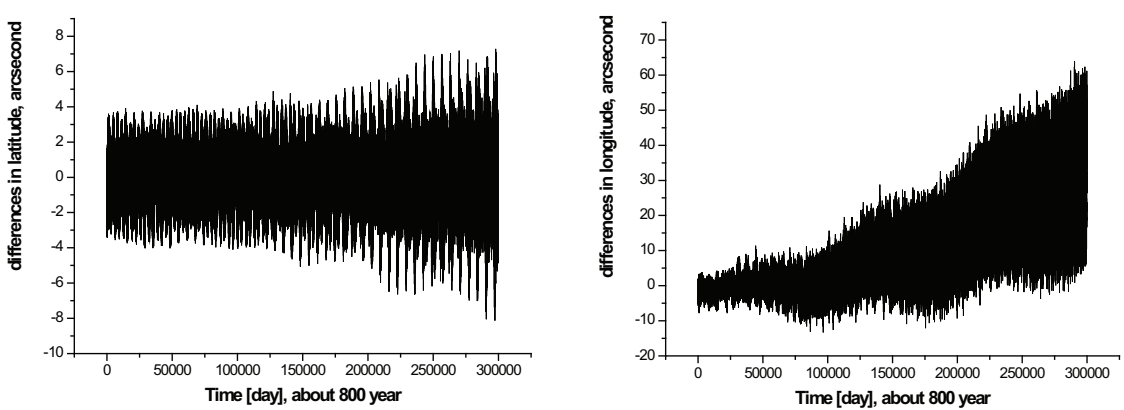

Рис. 6. Учет сжатия Земли и возмущений со стороны Венеры, слева приведена разность в широте, справа - в долготе

от сжатия Земли влияют в большей степени на широту, от Венеры - на долготу. Совместный учет обоих факторов уменьшает амплитуду разности в широте более чем вдвое и практически устраняет долгопериодические колебания с большой амплитудой разности в долготе.

\section{5 Выводы}

В предлагаемом исследовании дано краткое описание аналитической теории и численной теории с точки зрения методов их построения, учета возмущающих факторов и соответствующей точности. Достаточно подробно представлен алгоритм приведения обеих теорий к единой системе отсчета. Это приведение выполнено путем учета прецессии от планет, которая приводит к тому, что плоскость эклиптики за 8 столетий наклоняется на 400". Расчет положения центра масс Луны был проведен по обеим теориям на интервале в 800 лет. Результаты сравнения обеих теорий продемонстрированы на графиках. Мы также обнаружили, что если не принимать в расчет нелинейные слагаемые в аргументах Делоне, то вековое ускорение за 800 лет составит примерно $830^{\prime \prime}$ в остаточных разностях. Возмущения от Венеры и сжатия Земли приводят к существенным отличиям относительно главной проблемы с амплитудами соответственно около $14^{\prime \prime}$ и $8^{\prime \prime}$.

\section{Литература}

Абалакин В.К. // Основы эфемеридной астрономии. М.: Наука. 1979.

Гутцвиллер, Шмидт (Gutzwiller M.C., Schmidt D.S.) // Astronomical Papers prepared for the use of the American Ephemeris and Nautical Almanac. 1986. V. 23. P. 1.

Загидуллин и др. (Zagidullin A., Petrova N., Nefed'ev Yu.) // The Seventh Moscow Solar System Symposium 7M-S ${ }^{3}$. М.: ИКИ РАН. 2016. С. 225.

Лиске и др. (Lieske J.H., Lederle T., Fricke W., Morando B.) // Astron. Astrophys. 1977. V. 58. P. 1.

Петрова (Petrova N.) // Earth, Moon and Planets. 1996. V. 73. P. 71.

Петрова Н.К., Нефедьев Ю.А., Демин С.А., Гусев А.В. // Ученые записки Казанского университета. Физико-математические науки. 2013. Т. 155. Кн. 3. С. 19.

Пти, Лузум (Petit G., Luzum B.) // IERS Technical Note. 2010. N. 36. P. 1.

Симон и др. (Simon J.L., Bretagnon P., Chapront J., Chapront-Touzé M., Francou G., Laskar J.) // Astron. Astrophys. 1994. V. 282. P. 663.

Фолкнер и др. (Folkner W.M., Williams J.G., Boggs D.H., Park R.S., Kuchynka P.) // The Interplanetary Network Progress Report. 2014. V. 42-196C. P. 1.

Шапрон-Тузе, Шапрон (Chapront-Touzé M., Chapront J.) // Analytical ephemerides of the Moon in the 20th century. SYRTE - Observatoire de Paris. 2002. 